\title{
Impact of Early Urine Specimen Collection on Emergency Department Time to Disposition: A Randomized Controlled Trial
}

\author{
Amit Bahl ${ }^{1}$, Ameen M. Jamali ${ }^{2}$, Gautam Ramesh ${ }^{3}$ \\ 1. Emergency Medicine, Beaumont Hospital, Royal Oak, USA 2. Emergency Medicine, Medical Center Health System, \\ Odessa, USA 3. Emergency Medicine, Michigan State University College of Human Medicine, Lansing, USA
}

Corresponding author: Amit Bahl, ambahl79@gmail.com

\section{Abstract \\ Background}

Diagnostic testing in the ED increases the length of stay (LOS). Urinalysis testing is highlighted specifically as a source of delays. We aim to determine whether a triage-initiated urine specimen collection process decreases ED time to disposition (TTD) in ambulatory patients with abdominal pain.

\section{Methods}

This prospective, randomized controlled study was implemented at a Suburban Level One trauma ED with greater than 120,000 annual visits. A convenience sample of patients was recruited. Adult, non-ambulance patients presenting with abdominal pain were eligible. Participants were randomized into experimental and control groups. Patients in the control group provided a urine sample after physician evaluation, if ordered by the provider. Patients in the experimental group were prompted to provide a urine sample in the triage restrooms immediately after screening at the greeter desk. The UA sample was transported to the treatment area and sent to the laboratory after physician evaluation.

\section{Results}

A total of 125 control patients and 124 experimental patients were enrolled. Forty-two patients were excluded because they were unable to provide a urine sample. Patients who had a urinalysis ordered were included in statistical analysis. Final data set included 65 patients in the experimental group and 96 patients in the control group. No significant difference $(\mathrm{p}=0.5072)$ in disposition time between subjects in the experimental group $(n=65$, mean $=5: 17$ [hours:min]) and subjects in the control group $(n=96$, mean $=5: 30)$ was found.

\section{Conclusions}

Review began 09/10/2020 Review ended 09/13/2020 Published 09/16/2020

\section{() Copyright 2020}

Bahl et al. This is an open access article distributed under the terms of the Creative Commons Attribution License CC-BY 4.0., which permits unrestricted use, distribution, and reproduction in any medium, provided the original author and source are credited.
The triage protocol for urine specimen collection did not significantly reduce ED TTD. Further research in overcrowded EDs with long patient waiting room times may benefit from implementing a triage protocol for urine specimen collection.

Categories: Emergency Medicine, Quality Improvement

Keywords: ed length of stay, triage protocols, ed operations, urinalysis testing, urinalysis

\section{Introduction}

In the past 20 years, the number of visits to EDs has increased substantially. This increased number of visits has contributed to ED overcrowding nationwide [1-5]. ED overcrowding has been shown to increase the length of stay (LOS) and delay treatment [6-12]. Previous studies have identified the need for more front-end operations that can be used to decrease ED LOS and time to disposition (TTD) [13,14]. An example of such mechanism is a study by Singer et al., in which point-of-care troponin testing for patients with chest pain reduced TTD compared to patients whose troponin was measured in a central laboratory [15]. Another study investigated the impact of a triage order set for cardiac testing and found a similar reduction in TTD [16]. A third study found that a triage nurse $\mathrm{x}$-ray requesting system improves the flow of the walking wounded patients in the emergency room [17]. The previous studies are examples of utilization of specific front-end operations based on chief complaint and presentation. By implementing these operations, ED TTD decreases, ED LOS decreases, treatment delays are reduced, and patient outcomes are improved [18-24].

Because diagnostic tests increase patient's LOS, diagnostic testing time is a potential target for decreasing ED LOS [25]. In this study, urine specimen collection was investigated as a potential target for decreasing ED TTD. Urine specimen collection is patient-dependent and is highly variable in the amount of time it takes to complete. This study aimed to demonstrate that a simple triage protocol of urine specimen collection based 


\section{Materials And Methods}

\section{Study design}

This prospective, randomized controlled study of patients in an academic, suburban, Level One Trauma Center was conducted over a two-month period lasting from July 2014 to August 2014. Eligible participants were non-ambulance patients who were 21 years or older with a chief complaint of abdominal pain, flank pain, or pelvic pain. This study was approved by the home Institutional Review Board. Patients meeting eligibility were consented to participate in the study. Participants were randomized into experimental and control groups using an envelope system. Envelopes were prepared and sealed by a biostatistician prior to patient recruitment. The control group proceeded through the treatment process in traditional fashion, beginning at the greeter desk and culminating in the treatment area. Patients in the control group were asked to provide a urine sample after physical examination only if ordered by the provider. The treatment process of the experimental group started at the greeter desk, where patients received a urinalysis packet. Before proceeding to the treatment area, patients were prompted to provide a urine sample in the triage restrooms, which they carried with them to the treatment area. After physical examination, urine samples were sent for lab analysis, if ordered by the provider. Providers were unaware of patient recruitment into the study and were blinded to randomization arm.

Disposition order was placed into the electronic medical record by the physician, and was used as the primary outcome measure. Age, sex, heart rate, systolic blood pressure, labs, and imaging orders were examined as descriptive variables across control and experimental groups.

\section{Data collection and processing}

The patient's status (control or experimental group), chief complaint, medical records number (MRN), and date of ED visit were recorded in a password-protected Microsoft Excel spreadsheet. Additional information, including TTD, sex, age, heart rate, labs, imaging and disposition decision (discharged or admitted) was retrieved from the hospital's EMR system, Epic@ (Cary, NC).

\section{Outcome measure and analysis}

The primary outcome measured was TTD between treatment groups. TTD was used in order to prevent factors such as increased boarding times and prolonged discharges from skewing the data. A Wilcoxon twosample rank-sum test was conducted on the TTD between treatment groups using RStudio version 1.0.136 for Mac OS (RStudio, Boston, MA). Pearson chi-square and fisher's exact test were performed to analyze descriptive variables across treatment groups.

\section{Results}

Of the 249 patients recruited, 125 were randomized to the control group and 124 were randomized into the experimental group. 42 patients were excluded because they were unable to provide a urine sample leaving 121 in the control group, and 86 patients in the experimental group. Only patients who had a urinalysis ordered were included in statistical analysis. Final data set results included 65 patients in the experimental group and 96 patients in the control group, as shown in Figure 1. A Wilcoxon two-sample rank-sum test with Continuity Correction was performed on time to disposition in experimental versus control groups. No significant difference $(p=0.5072)$ in time to disposition between subjects in the experimental group $(n=65$, mean $=5: 17)$ and subjects in the control group ( $n=96$, mean=5:30 hours) was found. The primary outcome measure, time to disposition between experimental and control groups, is shown in Figure 2 and summarized in Table 1 . 


\section{Cureus}

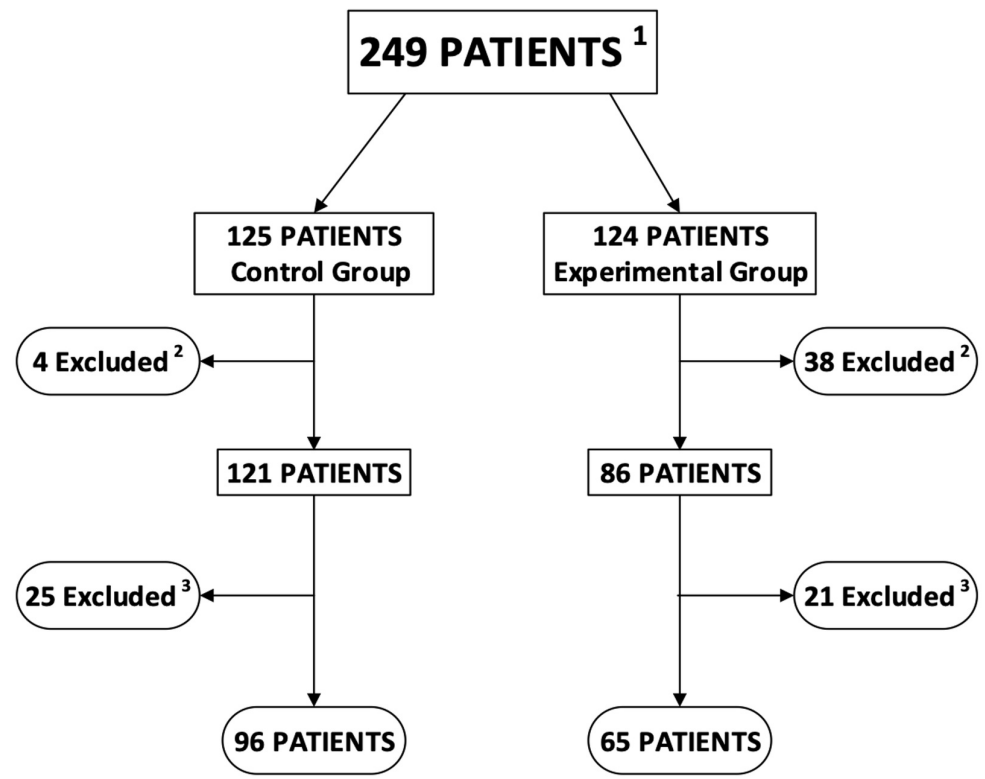

FIGURE 1: Diagram of patient flow

${ }^{1}$ Patients with chief complaint of abdominal pain and/or flank pain.

${ }^{2}$ Patients excluded as unable to provide urine sample in treatment pod bathroom in control group and triage bathroom in experimental group.

${ }^{3}$ Patients excluded as provider did not order urinalysis testing.

\begin{tabular}{|c|c|c|c|c|c|c|c|c|}
\hline Group & N & Mean & Std Dev & Minimum & Q1 & Median & Q3 & Maximum \\
\hline Experiment & 65 & $5: 17$ & 1:58 & 2:26 & 3:49 & 4:41 & 4:54 & 10:19 \\
\hline Control & 96 & $5: 30$ & 2:19 & $0: 37$ & 3:51 & 5:21 & $6: 51$ & 11:04 \\
\hline
\end{tabular}

TABLE 1: Primary outcome of time to disposition for experimental and control groups 


\section{Cureus}

Summary Statistics of Time to Disposition For Experimental and Control Groups

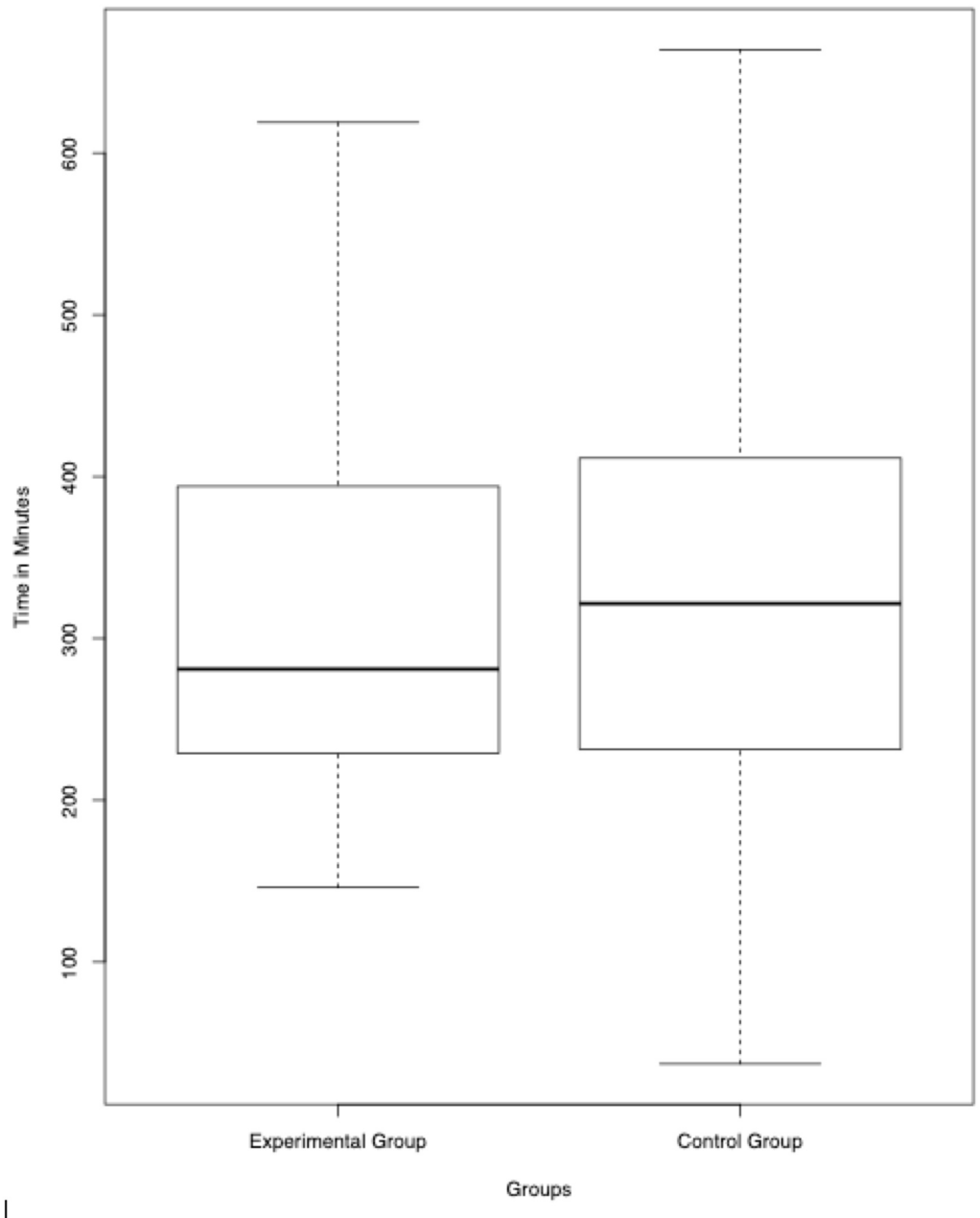

FIGURE 2: Time to disposition for experimental and control groups

A Wilcoxon two-sample rank-sum with continuity correction found no statistically significant difference between the time to disposition in the experimental and control groups $(p=0.5072)$.

Demographic information including age, gender, and vital signs were similar between groups. The average age was 46.41 in the control group and 42.85 in the experimental group ( $\mathrm{p}=0.1911)$. The majority $(78.12 \%)$ were female in the control group and $(75.68 \%)$ in the experimental group $(\mathrm{p}=0.3515)$. Table 2 shows full demographic statistics. 


\section{Cureus}

\begin{tabular}{|c|c|c|c|}
\hline \multicolumn{4}{|c|}{ Patient Characteristics } \\
\hline & Control $(n=96)$ & Experimental $(n=65)$ & p-value \\
\hline Mean Age & $46.41(17.7)$ & $42.85(16.3)$ & 0.1911 \\
\hline \multicolumn{4}{|l|}{ Gender } \\
\hline Male (\%) & $21(21.88 \%)$ & $19(29.69 \%)$ & \multirow[t]{2}{*}{0.3515} \\
\hline Female (\%) & $75(78.12 \%)$ & $45(75.68 \%)$ & \\
\hline Heart Rate & $86.5(15.6)$ & 87.7 (15.86) & 0.6408 \\
\hline Blood Pressure & $134.8(17.0)$ & $132.7(15.4)$ & 0.4273 \\
\hline \multicolumn{4}{|l|}{ Chief Com } \\
\hline Abdominal Pain & $85(88.54 \%)$ & $52(80.0 \%)$ & \multirow{2}{*}{0.1761} \\
\hline Flank Pain & $11(11.45 \%)$ & $13(20.00 \%)$ & \\
\hline \multicolumn{4}{|l|}{ Disposition } \\
\hline Admission & $50(52,08 \%)$ & $29(44.62 \%)$ & 0.4417 \\
\hline Discharge & $46(47.92 \%)$ & $36(55.38 \%)$ & \\
\hline \multicolumn{4}{|l|}{ Imaging } \\
\hline Yes & $87(90.62 \%)$ & 56 (86.15\%) & 0.5297 \\
\hline No & . $38 \%)$ & 9 (13.85\%) & \\
\hline
\end{tabular}

TABLE 2: Patient demographic and clinical variables

Patients in both groups had similar final disposition from the ED and similar amount of imaging ordered. Approximately half of the study subjects were admitted to the inpatient wards in both groups with $52.08 \%$ and $44.62 \%$ of patients in the control and experimental arms respectively being hospitalized ( $\mathrm{p}=0.4417)$. Imaging was similar between both groups with $90.62 \%$ and $86.15 \%$ in the control and experimental groups, respectively, receiving imaging (Table 2).

\section{Discussion}

This study demonstrated that early collection of urine in an ambulatory ED population with abdominal pain did not improve the time to disposition. Contrary to our hypothesis, early collection of a urine sample in triage did not positively impact our ED LOS. Overall ED operational efficiency at our site likely influenced this outcome measure. Despite significant daily volumes, our ED is highly efficient with a "straight back" process so that patients spend very little time in the triage bay. Instead, patients are taken directly to the treatment bays and are quickly assessed by nursing teams and physician providers. The average door-todoctor time was 25 minutes during our study period. The impact of the early urinalysis collection intervention may have been more relevant in a hospital with more ED overcrowding with longer patient wait times. Recreation of this study in an overcrowded ED, along with recording ED volume at the time of patient recruitment, could allow the effect of this triage urine specimen collection to be evaluated more thoroughly.

Testing in the ED has a significant impact on ED LOS. In a study by Kocher et al. analyzing 360 million ED visits, $63 \%$ of patients received some level of testing. Blood tests which included urinalysis for the purpose of this study when performed were associated with an odds ratio of 2.29 for experiencing more than a four-hour ED LOS. The most time-costly testing modalities included blood testing as the leader of the group. Optimizing delivery of emergency care requires a closer analysis of testing protocols to improve operational efficiency [25].

Front-end operational interventions such as this study have the potential to decrease ED LOS and improve the overall quality of care with downstream implications of reducing ED overcrowding and improved patient outcomes and satisfaction [18-24,26-29]. Urinalysis was chosen for this study as this data point has the potential for significant delay as it is impacted by the patient's ability to void compared to other blood testing that is largely dependent on the practitioner taking the blood sample [25]. We felt that emphasizing the need for a urine specimen early in the ED visit would improve the time to urine collection. We were unable to find other studies evaluating early urine collection and the impact on ED LOS. Some literature 
exists regarding point-of-care testing with urine dipstick as a means to improve the efficiency of urinalysis testing but poor sensitivities and specificities to diagnose urinary tract infection by dipstick in several studies may limit the benefit of this type of testing [30]. Further, this intervention addresses time of testing but not the time to acquire the sample from the patient, the interval of interest in this study. Also, the pointof-care testing requires additional resources and training.

Our study was not without some limitations. The study included only non-ambulance patients as most patients arriving via EMS do not spend time in triage at our institution. Thus the impact of a similar intervention on ambulance traffic is unclear. As these patients may have a higher severity of illness with higher admission rates, it is possible that the results of our analysis would be different. Further, we narrowed our evaluation to exclude patients with altered mental status or fever. As urinary tract infection in the elderly is a common cause of both of these complaints, early collection of urinalysis may impact ED disposition times in these subgroups. As many of these patients arrive via EMS, they were excluded from this analysis.

We recruited a convenience sample of patients on weekdays from 9 am to $5 \mathrm{pm}$. We did not analyze trends in ED volumes during recruitment or consider differences in operational efficiencies during "non-business" hours. It is unclear but our results would have been impacted by a different recruitment schedule.

Another limitation was that urinalysis was not submitted to the laboratory until the physician ordered the test rather than laboratory submission immediately after triage collection in the experimental group. While this was an extremely common test ordered in patients with abdominal pain, not all patients (approximately $20 \%$ in our investigation) required the test and we felt the decision was best left to the treating physician. More prompt laboratory submission for all patients in the experimental group may have resulted in shorter urinalysis result times and reduced ED LOS.

A large number of patients were excluded from the analysis as a significant number of patients were unable to provide a urine sample or the provider chose not to order urinalysis testing. While the two comparative groups appeared to be similar as depicted in the analysis, the impact on the time to disposition for the randomized but excluded patients particularly on the experimental side was unknown.

\section{Conclusions}

The triage protocol for urine specimen collection described in this study did not significantly reduce ED time to disposition. Further research in overcrowded EDs with longer patient waiting room times with patients with a more diverse range of chief complaints may provide better insight into which hospitals and populations may benefit from implementing a triage protocol for urine specimen collection.

\section{Additional Information}

\section{Disclosures}

Human subjects: Consent was obtained by all participants in this study. Beaumont Health Institutional Review Board issued approval 2013-178. This study was approved by the Institutional Review Board. Patients meeting eligibility were consented to participate in the study. . Animal subjects: All authors have confirmed that this study did not involve animal subjects or tissue. Conflicts of interest: In compliance with the ICMJE uniform disclosure form, all authors declare the following: Payment/services info: All authors have declared that no financial support was received from any organization for the submitted work. Financial relationships: All authors have declared that they have no financial relationships at present or within the previous three years with any organizations that might have an interest in the submitted work. Other relationships: All authors have declared that there are no other relationships or activities that could appear to have influenced the submitted work.

\section{References}

1. Committee on the Future of Emergency Care in the United States Health System. Hospital-based Emergency Care: At the Breaking Point. Washington DC: National Academies Press; 2006.

2. Asplin BR: Tying a knot in the unraveling health care safety net . Acad Emerg Med. 2001, 8:1075-1079. 10.1111/j.1553-2712.2001.tb01118.x

3. Kellerman AL: Crisis in the emergency department. N Engl J Med. 2006, 355:1300-1303. 10.1056/NEJMp068194

4. Nawar EW, Niska RW, Xu J: National Hospital Ambulatory Medical Care Survey- 2005 emergency department survey: advance data for vital health statistics, number 386, June 29. 2007. CDC Website. Available at: http://www.cdc.gov/nchs/data/ad/ad386.pdf, 4:2015.

5. Approaching full capacity. American College of Emergency Physicians Website. Available at: http://www.acep.org/workarea/downloadasset.aspx?id=8852. Accessed June 5. (2015). http://www.acep.org/workarea/downloadasset.aspx?id=8852..

6. Slade EP, Dixon LB, Semmel S: Trends in the duration of emergency department visits, 2001-2006. Psychiatr Serv. 2010, 61:878-884. 10.1176/ps.2010.61.9.878

7. Herring A, Wilper A, Himmelstein DU, Woolhandler S, Espinola JA, Brown DFM, Camargo Jr CA: Increasing 
length of stay among adult visits to U.S. emergency departments, 2001-2005. Acad Emerg Med. 2009, 16:609-616. 10.1111/j.1553-2712.2009.00428.x

8. Forster AJ, Steill I, Wells G, et al.: The effect of hospital occupancy on emergency department length of stay and patient disposition. Acad Emerg Med. 2003, 10:127-133. 10.1111/j.1553-2712.2003.tb00029.x

9. Hollander JE, Pines JM: The emergency department crowding paradox: the longer you stay, the less care you get. Ann Emerg Med. 2007, 50:497-499. 10.1016/j.annemergmed.2007.05.002

10. Schull MJ, Vermeulen M, Slaughter G, et al.: Emergency department crowding and thrombolysis delays in acute myocardial infarction. Ann Emerg Med. 2004, 44:577-585. 10.1016/j.annemergmed.2004.05.004

11. Hwang U, Richardson LD, Sonuyi TO, et al.: The effect of emergency department crowding on the management of pain in older adults with hip fracture. J Am Geriatr Soc. 2006, 54:270-275. 10.1111/j.15325415.2005.00587.x

12. Chen EH, Mills AM, Lee BY, et al.: The impact of a concurrent trauma alert evaluation on time to head computed tomography in patients with suspected stroke. Acad Emerg Med. 2006, 13:349-352. 10.1197/j.aem.2005.10.011

13. Gregory CJ, Marcin JP: Golden hours wasted: the human cost of intensive care unit and emergency department inefficiency. Crit Care Med. 2007, 35:1614-1615. 10.1097/01.CCM.0000266826.34532.FD

14. Retezar R, Bessman E, Ding R: The effect of triage diagnostic standing orders on emergency department treatment time. Ann Emerg Med. 2011, 57:89-99. 10.1016/j.annemergmed.2010.05.016

15. Singer AJ, Ardise J, Gulla J, Cangro J: Point-of-care testing reduces length of stay in emergency department chest pain patients. Ann Emerg Med. 2005, 45:587-591. 10.1016/j.annemergmed.2004.11.020

16. Hwang CW, Payton T, Weeks E, Plourde M: Implementing triage standing orders in th emergency department leads to reduced physician-to-disposition times. Adv Emerg Med. 2016, 2016:7213625. $10.1155 / 2016 / 7213625$

17. Lindley-Jones M, Finlayson BJ: Triage nurse requested xrays -are they worthwhile? . Emerg Med J. 2000, 17:103-107. 10.1136/emj.17.2.103

18. Polevoi SK, Quinn JV, Kramer NR: Factors associated with patients who leave without being seen . Acad Emerg Med. 2005, 12:232-236. 10.1197/j.aem.2004.10.029

19. Rodi SW, Grau MV, Orsini CM: Evaluation of a fast track unit: alignment of resources and demand results in improved satisfaction and decreased length of stay for emergency department patients. Qual Manag Health Care. 2006, 15:163-170. 10.1097/00019514-200607000-00006

20. Boudreaux ED, D'Autremont S, Wood K, et al.: Predictors of emergency department patient satisfaction: stability over 17 months. Acad Emerg Med. 2004, 11:51-58. 10.1197/j.aem.2003.06.012

21. Magid DJ, Sullivan AF, Cleary PD, et al.: The safety of emergency care systems: results of survey of clinicians in 65 US emergency departments. Ann Emerg Med. 2009, 53:715-723. 10.1016/j.annemergmed.2008.10.007

22. Pines JM, Hollander JE: Emergency department crowding is associated with poor care for patients with severe pain. Ann Emerg Med. 2008, 51:1-5. 10.1016/j.annemergmed.2007.07.008

23. Sprivulis PC, Da Silva JA, Jacobs IG, et al.: The association between hospital overcrowding and mortality among patients admitted via Western Australian emergency departments. Med J Aust. 2006, 184:208-212.

24. Richardson DB: Increase in patient mortality at 10 days associated with emergency department overcrowding. Med J Aust. 2006, 184:213-216.

25. Kocher KE, Meurer WJ, Desmond JS, Nallamothu BK: Effect of testing and treatment on emergency department length of stay using a national database. Acad Emerg Med. 2012, 19:525-534. 10.1111/j.15532712.2012.01353.x

26. Gardner RL, Sarkar U, Maselli JH, Gonzales R: Factors associated with longer ED lengths of stay . Am J Emerg Med. 2007, 25:643-650. 10.1016/j.ajem.2006.11.037

27. Hoot NR, Aronsky D: Systematic review of emergency department overcrowding: causes, effects, and solutions. Ann Emerg Med. 2008, 52:126-136. 10.1016/j.annemergmed.2008.03.014

28. Fee C, Weber EJ, Maak CA, et al.: Effect of emergency department crowding on time to antibiotics in patients admitted with community-acquired pneumonia. Ann Emerg Med. 2007, 50:501-509. 10.1016/j.annemergmed.2007.08.003

29. Urgent Matters Program. Perfecting patient flow: America's safety net hospitals and emergency department crowding. Washington, DC: National Association of Public Hospitals and Health. Systems. 2005,

30. Propp DA, Weber D, Ciesla ML: Reliability of a urine dipstick in emergency department patients . Ann Emerg Med. 1989, 5:560-563. 10.1016/s0196-0644(89)80844-3 